\title{
Disorders of the spine. A major health and social problem
}

\author{
Filip Raciborski ${ }^{1}$, Robert Gasik², Anna Kłak ${ }^{1}$
}

${ }^{1}$ Department of Gerontology and Public Health, National Institute of Geriatrics, Rheumatology and Rehabilitation, Warsaw, Poland ${ }^{2}$ Department of Neuro-orthopaedics and Neurology, National Institute of Geriatrics, Rheumatology and Rehabilitation, Warsaw, Poland

\begin{abstract}
According to the data published in The Lancet, in 2010 musculoskeletal disorders were the cause of nearly 166 million years lived with disability (YLDs), with neck and low back pain accounting for $69.9 \%$ of the total. In Poland, in 2014 low back pain was self-reported by $28.4 \%$ of women and $21.2 \%$ of men aged 15 years and over, neck pain by $21 \%$ of women and $13 \%$ of men, and middle back pain by $19 \%$ of women and $12.9 \%$ of men. In 2015 , nearly 33 million man-days were lost due to spine disorders, and nearly 2.7 million medical certificates were issued for back pain (15\% of the total). With the current demographic changes (population ageing) and lifestyle-related factors increasing the potential for back problems, the demand for a wide range of medical services to treat disorders of the spine and their symptoms may be expected to increase substantially over the coming years.
\end{abstract}

Key words: spine, low back pain, epidemiology, sickness absenteeism from work.

\section{Introduction}

Spine disorders (SD) and the associated pain are a major medical, social and economic problem because of their high prevalence and the ever-increasing number of patients with SD in the general population [1]. A delay between the first symptoms and the diagnosis of SD and onset of treatment with a potentially adverse impact on treatment outcome is a major concern. Another is the lack of effective prevention which would reduce the incidence of SD. Additionally, there are social and economic consequences of SD as many people with back problems leave the labour market, either temporarily or permanently, usually with adverse effects on their family life and socio-economic status.

\section{Etiology}

In most cases of SD, patients experience pseudoradicular pain [2] and in the majority the pain results from degenerative changes in the spine, involving the intervertebral discs, facet joints and soft tissues. Less common causes of pseudoradicular pain include infections, non-infectious inflammation, tumours and metabolic disease (e.g. osteoporosis). The second most common cause of back pain is compression of a spinal nerve root (radicular pain). Radicular pain may also occur in association with degenerative changes, infections, rheumatic and metabolic diseases, tumours and injuries.

A degenerative disease of the spine is the major cause of back pain [3]. The first symptoms are experienced in the third and fourth decades of life. The pain usually quickly subsides, but depending on the location and severity of degenerative changes it may become chronic. Such chronic pain may be reported by up to $60 \%$ of patients. According to the Central Statistical Office of Poland (GUS), in 2007 back pain was the main reason for consulting general practitioners.

In a degenerative disease of the spine, an intervertebral disc (ID) is the first structure affected with a loss of height and decreased content of proteoglycans and water in the annulus fibrosus and the nucleus pulposus. As a result, discogenic pain may develop [4]. Discogenic pain felt in the paraspinal region is diffuse and exacerbated by movement, and may persist for a few days to a few weeks. Degenerative changes in the ID(s) may produce pain in the facet joints. That pain is associated with the increased pathological range of motion in the spine and increased forces acting on the joints caused by the reduc-

\footnotetext{
Address for correspondence:

Anna Kłak, Department of Gerontology and Public Health, National Institute of Geriatrics, Rheumatology and Rehabilitation, Spartanska 1, 02-637 Warsaw, Poland, e-mail: klak.anna@o2.pl
}

Submitted: 22.07.2016, Accepted: 12.08.2016 
Table I. Disease entities characterized by specific warning signs and symptoms

\begin{tabular}{|ll|}
\hline Warning symptoms and signs & Disease entity \\
\hline $\begin{array}{l}\text { Back pain associated with elevated body tem- } \\
\text { perature and weight loss }\end{array}$ & $\begin{array}{l}\text { infectious discitis, non-infectious discitis, spinal epidural abscess, tuberculo- } \\
\text { sis, brucellosis, fungal infections, Wegener's granulomatosis }\end{array}$ \\
\hline Nocturnal back pain & $\begin{array}{l}\text { tumours of nervous and bone structures of the spine, vascular malforma- } \\
\text { tions, syringomyelia }\end{array}$ \\
\hline Back pain associated with morning stiffness & $\begin{array}{l}\text { ankylosing spondylitis, rheumatoid arthritis, spinal stenosis with neurogenic } \\
\text { claudication }\end{array}$ \\
\hline
\end{tabular}

tion in the size of the ID. Also, degenerative ID changes may cause a tear in the annulus fibrosus and bulging of the nucleus pulposus into the spinal canal. The resulting compression of the neural structures may produce neurological symptoms corresponding to its site. Spinal stenosis, congenital or acquired, may be associated with radicular pain or pain due to decreased flow in the microcirculation of the nerve roots and spinal nerves. Acquired stenosis results from the degenerative changes in the intervertebral discs and facet joints which narrow the space for the neural structures. Neurogenic claudication is characterized by sensory neurological symptoms (e.g. paraesthesia, superficial sensation deficits) and muscular weakness in the lower extremities exacerbated by walking. Spine disorders may also be associated with pain felt in e.g. sacroiliac or hip joints. In some cases the pain only mimics spine disorders. Frequently, pain originating in the sacroiliac joints is felt in the low back and is considered a symptom of spine disorders. Also, disorders of the spine such as pathological curvature of the spine may lead to an excessive strain on the joints of the lower extremities with the resulting changes and joint pain.

When examining symptoms caused by different spine disorders it is necessary to consider cancer, infectious inflammation of spinal structures and fractures (Table I) [5]. In such cases the signs and symptoms, known as red flags, are indicators of serious conditions in which an accurate and prompt diagnosis followed by timely treatment is required (Table II).

\section{Epidemiology}

The knowledge of the epidemiology of spine disorders and back pain is limited in part due to methodology issues. Often, respondents report a spine disorder as low back pain irrespective of the actual diagnosis or cause of the condition [6].

According to last week's Eurobarometer survey, muscle, bone and joint pain restricting the activities of daily living (ADLs) occurred in almost every third adult (32\%) living in Europe. The percentage varied between countries and ranged from $18 \%$ in Ireland to $46 \%$ in Croatia. In Poland, activity-limiting pain was reported by $39 \%$ of the respondents. Low-back pain was the most common complaint, reported by $11 \%$ of all respondents, followed by pain in the upper back (8\%) and neck (7\%) [7]. Pain was nearly twice as common in older people (aged 55 years and over) than in the youngest age group (15 to 24 year olds), $43 \%$ and $23 \%$ respectively. There were also differences by gender, with pain reported by $37 \%$ of women and $27 \%$ of men [7].

Nearly every fourth respondent (25\%) in the Eurobarometer survey reported at least one incident of muscle, bone and joint pain lasting three months or longer in their lifetime. The lowest rate was reported from Greece (13\%) and the highest from Austria (35\%). The rate in Poland was at the average European prevalence level, with 25\% of Polish respondents reporting at least one episode of chronic joint, bone and muscle pain in their lifetime. Most of them (9\%) described low back pain, followed by upper back (6\%) and neck (5\%) pain. Chronic pain was more frequently reported by women (28\%) than by men (22\%) [7].

Hoy et al. [8] estimated the prevalence of low back pain from the Global Burden of Disease 2010 study. In 2010, the global age-standardized prevalence was 9.4\%, with a higher rate in men (10.1\%) than in women (8.7\%). In Western Europe the prevalence of low back pain was $15.5 \%$ in men and $14.5 \%$ in women. The corresponding figures were $12.6 \%$ vs. $10.3 \%$ in Central Europe and $12.2 \%$ vs. 10.4\% in Eastern Europe. The prevalence increased with age, reaching a peak around the age of 80 years, and then slightly decreased [8].

Table II. Red flags for serious spine disorders

\begin{tabular}{|l|}
\hline Red flags for serious spine disorders \\
\hline First incident of back pain after the age of 50 years or \\
before the age of 20 \\
Back pain increasing in severity at night \\
Back pain increasing in severity in the lying position and not \\
related to physical activity \\
Diagnosed cancer \\
Immunosuppression \\
Neurological deficit confirmed by physical examination \\
- sphincter dysfunction \\
- muscle weakness \\
- impaired superficial sensation, especially in the pubic area
\end{tabular}




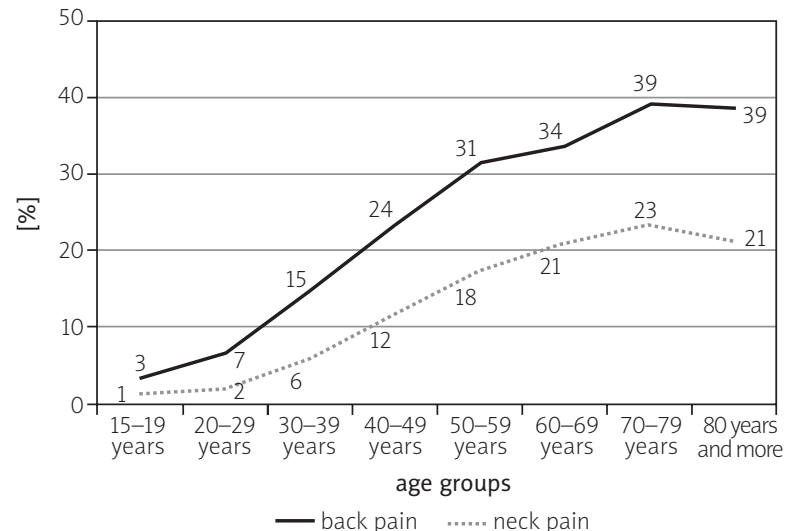

Fig. 1. Chronic diseases and complaints in Poland: back pain and neck pain by age. EHIS (2009).

Source: [12]

Hoy et al. also conducted a systematic review of a total of 165 published studies from 54 countries. The mean prevalence of low back pain was 31\%. After adjusting for the duration of pain, the mean point prevalence was estimated to be $11.9 \%$ and the 1-month prevalence was estimated to be 23.2\% [9].

The Global Burden of Disease 2010 study also estimated the prevalence of neck pain. The global prevalence was $4.9 \%$, with a higher rate in women than in men (5.8\% vs. $4.0 \%)$. In Western Europe, the prevalence of neck pain was $5.2 \%$ in men and $7.4 \%$ in women. The corresponding figures were $4.1 \%$ vs. $5.8 \%$ in Central Europe and $4.0 \%$ vs. $5.9 \%$ in Eastern Europe. The prevalence of neck pain was age-related and increased to the age of about 45 years in both men and women and then substantially decreased in older subjects [10].

According to the preliminary findings from the European Health Interview Survey (EHIS) conducted in 2014, $59 \%$ of adult respondents in Poland reported health problems or chronic disease, low back pain being the most common complaint. It was reported by $28.4 \%$ of females and $21.2 \%$ of males aged 15 years and over and was followed by neck pain (21\% of women and $13 \%$ of men) and middle back pain (19\% of women and $12.9 \%$ of men). To compare, high blood pressure was reported by $25.7 \%$ of women and $20.7 \%$ of men [11].

Detailed information about the prevalence of diseases and pain disorders is not yet available from the EHIS 2014, and it is necessary to refer to the earlier findings (EHIS 2009). In the earlier study, the respondents were asked about back pain in general, not about its specific location. Both back and neck pain was more common in older age groups, the only exception being neck pain in people aged 80 years and over [12]. Figure 1 presents the prevalence rates.

\section{Trends: what will the health situation be in Poland in 2050?}

The prevalence of back pain and spine disorders increases with age. According to the EHIS 2009 findings published by the Central Statistical Office of Poland (GUS), almost every fourth respondent aged 40-49 years (24\%) and every third respondent aged 60-69 years $(34 \%)$ reported chronic back pain, compared to just 3\% of 15-19-year-olds [12]. According to the GUS demographic data, the population of Poland is ageing rapidly. In 1990, the median age was 33.7 years for women and 30.9 years for men. Nearly a quarter of a century later (in 2013), the respective figures were 40.9 years and 37.4 years. In 2050, the median age of 54.8 years for women and 50.1 years for men is predicted [13]. This increase in the median age is related to, among other factors, increased average life expectancy. Between 1991 and 2013, the average life expectancy in Poland increased by 6 years in women and by 7.2 years in men, to 81.1 years and 73.1 years respectively [13].

These changes will be reflected in the size of the population in particular age groups. The number of people aged 40-59 years will increase by 1.1 million by the year 2030 (compared to 2013) to 11.5 million and subsequently will decrease to 8 million by 2050 . By 2025 , the number of 60-79-year olds will increase by 1.9 million to 8.7 million. These figures would remain stable for a period of time followed by another increase up to 10.2 million people in 2050. In the years 2013-2050, the number of people aged 80 years and over will increase from 1.5 million to 3.5 million (Fig. 2). In Poland, by the year 2035 the number of people aged 40 years and over will increase by over 3.8 million compared to 2013, i.e. by nearly $21 \%$. This will translate into a significantly increased demand for a wide range of medical services to manage disorders of the spine and back pain.

\section{Burden of spine disorders and absenteeism}

According the Global Burden of Disease Study 2010 published in The Lancet, musculoskeletal disorders are the second main cause, after mental and behavioural disorders, of disability worldwide measured by years lived with disability (YLDs). Low back pain was the leading specific contributor to YLDs [14]. Vos et al. [14] estimated that in 2010 musculoskeletal disorders accounted worldwide for nearly 166 million YLDs (95\% Cl: 9126.3637-208.7785) with back and neck pain making up $69.9 \%$ of the total ( $95 \% \mathrm{Cl}: 80.6151-156.5268)$. According to the analysis of Murray et al. [15], low back pain was the sixth out of 291 causes of the global burden of disease measured by disability-adjusted life years (DALY). In Western Europe it is the first cause and 


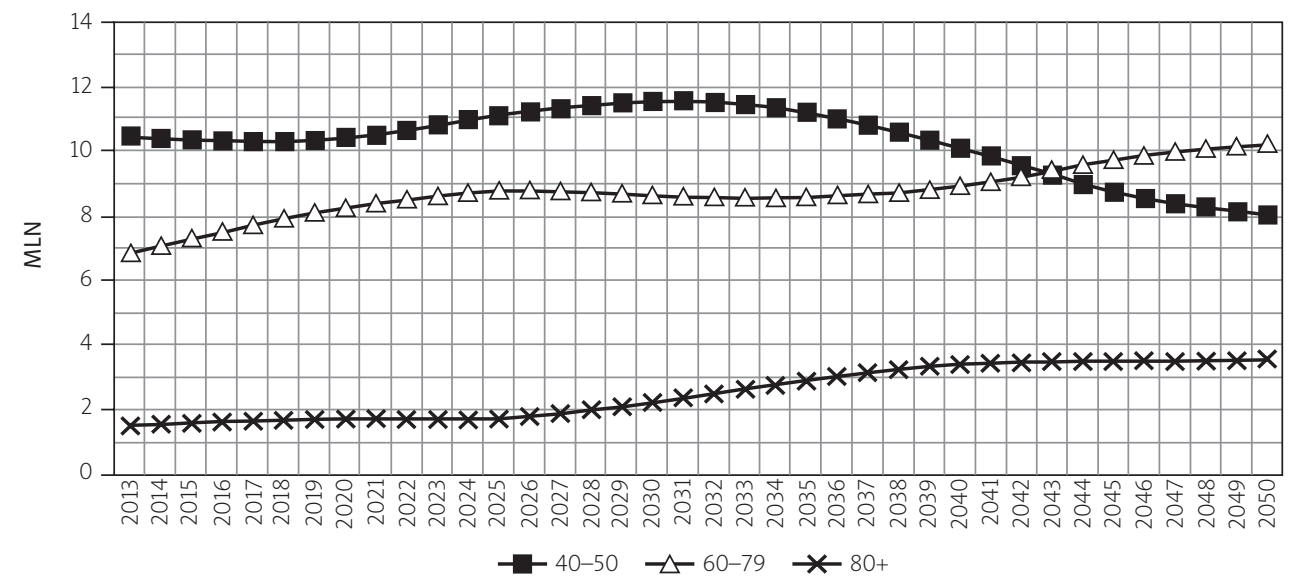

Fig. 2. Predicted changes in the population size in specific age groups in Poland in the years 2013-2050. Source: Own analysis based on the GUS data.

in Central and Eastern Europe the third cause of healthy life-years lost. Neck pain is the 21st most important contributor to the global disease burden (the number 8 cause in Western Europe, number 14 in Central Europe and number 18 in Eastern Europe) [15].

Spine disorders and back pain also have a significant impact on ability to work. According to Poland's Social Insurance Institution (ZUS) data, in 2014 disorders of the spine were the cause of nearly 31 million days of absence from work due to illness, accounting for 15\% of all medical certificates. In 2015, absence from work due to spine disorders was nearly 33 million man-days, an increase by 2 million from 2014. However, the total number of days of absence from work recorded by ZUS increased in that period from 213 million to 227 million man-days. In 2015, nearly 2.7 million medical certificates (15\% of all medical certificates) were issued for spine disorders [16].

\section{Treatment}

Appropriate management of spine disorders requires an interdisciplinary approach with the participation of health care professionals representing different specialties, including a rheumatologist, a physiotherapist, a surgeon, a specialist in the treatment of osteoporosis and a psychologist. Such an approach is guided by the varied etiology of most common painful disorders of the spine such as degenerative disease, injuries or strain. Pharmacological treatment is the mainstay and focuses mostly on adequate pain relief in the period of symptom worsening. The pharmacological treatment of pain should be consistent with the World Health Organization (WHO) analgesic ladder, while the response to analgesics is to some degree limited by the anatomy of the spine, e.g. the intervertebral disc tissues are avascular after the age of 2. The pharmacological treatment should also target the underlying disease, e.g. osteoporosis.

Rehabilitation is another step of treatment, and physiotherapy is an important complement to the primary pharmacological treatment of chronic painful disorders of the spine. Special procedures are also used for controlling chronic pain such as nerve blocks, neurodestruction, neurostimulation and neuromodulation. When the conservative treatment fails, surgery, either conventional or minimally invasive, should be performed, which is also advisable in the case of focal neurological signs.

\section{Summary}

Disorders of the spine are an important health and social problem, as they are one of the main contributors to disability worldwide. Almost one in seven medical certificates in Poland is issued for back problems. A rise in the elderly population means that the number of patients requiring a range of medical services for the management of spine disorders will substantially increase. Considering the low level of physical activity in the population, obesity and sedentary lifestyle, it is essential to develop a prevention strategy focused on spine disorders and back pain. The programme should involve the entire population, but its components should be designed specifically for particular social groups.

In the first place, it is necessary to:

- raise awareness about spine disorders and their prevention amongst the general public, through health education and promotions also in collaboration with the media,

- develop new solutions for effective prevention of spine disorders, 
- initiate collaboration with specialists in occupationa medicine and primary care physicians to develop new solutions for early diagnosis, treatment and rehabilitation of spine disorders and painful back problems,

- develop guidelines for the management of spine disorders in primary care,

- develop guidelines for proper ergonomics of the workplace for employers and employees,

- develop guidelines for proper ergonomics in schools and universities,

- develop simple and easy-to-understand principles of spine disorder prevention for different social groups ("Spinal health tips"),

- raise awareness about spine disorders amongst public policy decision-makers, including the Minister of Health, Minister for Family, Labour and Social Policy, Minister of Education and members of the Sejm and Senate (Parliament) and initiate a search for novel system solutions.

The social campaign "Healthy spine for Poland" is an example of a project to implement the above proposals. The project is managed by an interdisciplinary team of experts consisting of representatives of patient associations, physicians and research workers concerned with the prevention and treatment of spine disorders.

The authors declare no conflict of interest.

\section{References}

1. Coenen P, Smith A, Paananen M, et al. Trajectories of low-back pain from adolescence to young adulthood. Arthritis Care Res (Hoboken) 2016; doi: 10.1002/acr.22949. Epub ahead of print.

2. Van Boxem K, Van Zundert J, Van Zundert J, et al. Pseudoradicular and radicular low-back pain: How to diagnose clinically? Pain. 2008; 135: 311-312; (author reply 313-315).

3. de Bruin F, ter Horst S, Bloem HL, et al. Prevalence of degenerative changes of the spine on magnetic resonance images and radiographs in patients aged 16-45 years with chronic back pain of short duration in the Spondyloarthritis Caught Early (SPACE) cohort. Rheumatology (Oxford) 2016; 55: 56-65.

4. Verrills P, Nowesenitz G, Barnard A. Prevalence and Characteristics of Discogenic Pain in Tertiary Practice: 223 Consecutive Cases Utilizing Lumbar Discography. Pain Med 2015; 16: 1490-1499.

5. Hooten WM, Cohen SP. Evaluation and Treatment of Low Back Pain: A Clinically Focused Review for Primary Care Specialists. Mayo Clin Proc 2015; 90: 1699-1718.

6. EUMUSC.NET. Musculoskeletal Health in Europe. http://www. eumusc.net/myUploadData/files/30\%20August\%20Final\%20 draft\%20report.pdf [accessed 10 April 2016].

7. European Commission 2007. Health in the European Union. Special Eurobarometer 272. 2007. http://ec.europa.eu/health/ ph_publication/eb_health_en.pdf [accessed 10 April 2016].

8. Hoy D, March L, Brooks P, et al. The global burden of low back pain: estimates from the Global Burden of Disease 2010 study.
Ann Rheum Dis 2014; 73: 968-974. doi: 10.1136/annrheumdis-2013-204428.

9. Hoy D, Bain C, Williams G, et al. A systematic review of the global prevalence of low back pain. Arthritis Rheum 2012; 64: 2028-2037.

10. Hoy D, March L, Woolf A, et al. The global burden of neck pain: estimates from the global burden of disease 2010 study. Ann Rheum Dis 2014; 73: 1309-1315.

11. Central Statistical Office of Poland. Health and health behaviour among the inhabitants of Poland according to the European Health Interview Survey (EHIS) 2014. Information note.

12. Central Statistical Office of Poland - Population Health Status 2009. Warsaw 2011

13. Central Statistical Office of Poland. Population Projection 2014-2050, Warszawa 2014

14. Vos T, Flaxman AD, Naghavi M, et al. Years lived with disability (YLDs) for 1160 sequelae of 289 diseases and injuries 19902010: a systematic analysis for the Global Burden of Disease Study 2010. Lancet 2012; 380: 2163-2196.

15. Murray CJ, Vos T, Lozano R, et al. Disability-adjusted life years (DALYs) for 291 diseases and injuries in 21 regions, 1990-2010: a systematic analysis for the Global Burden of Disease Study 2010. Lancet 2012; 380: 2197-2223.

16. ZUS Statistical Portal. http://psz.zus.pl/ [accessed 14 April 2016]. 